\title{
Antitumor effect of resveratrol on chondrosarcoma cells via phosphoinositide 3-kinase/AKT and p38 mitogen-activated protein kinase pathways
}

\author{
ZIXUN DAI, PENGFEI LEI, JIE XIE and YIHE HU \\ Department of Orthopedics, Xiangya Hospital of Central South University, Changsha, Hunan 410008, P.R. China
}

Received July 7, 2014; Accepted March 23, 2015

DOI: $10.3892 / \mathrm{mmr} .2015 .3683$

\begin{abstract}
Chondrosarcoma is one of the most common types of primary bone cancer that develops in cartilage cells. Resveratrol (Res), a natural polyphenol compound isolated from various fruits, has a suppressive effect on various human malignancies. It has been shown that Res inhibits matrix metalloproteinase (MMP)-induced differentiation in chondrosarcoma cells. However, the effects of Res on cell proliferation, apoptosis and invasion of chondrosarcoma cells, as well as the underlying mechanisms, remain largely unknown. To the best of our knowledge, the present study showed for the first time that Res inhibited proliferation and induced apoptosis in chondrosarcoma cells in a dose-dependent manner. Furthermore, it was shown that Res also suppressed chondrosarcoma cell invasion in a dose-dependent manner, probably via the inhibition of MMP2 and MMP9 protein expression. Molecular mechanism investigations revealed that Res could inhibit the activity of phosphoinositide 3-kinase/AKT and p38 mitogen-activated protein kinase signaling pathways, which has been demonstrated to be important in the regulation of proliferation, apoptosis and invasion in various cancer cell types. In conclusion, this study suggests that Res may serve as a promising agent for the treatment of chondrosarcoma.

\section{Introduction}

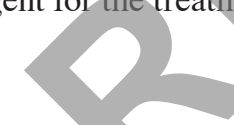

Chondrosarcoma is one of the most common types of primary bone cancer that develops in cartilage cells (1). Surgical resection is the most successful method to treat chondrosarcoma. As the effects of adjuvant treatments, such as proton beam radiation and chemotherapy are limited, the prognosis of patients with chondrosarcoma remains poor $(1,2)$. Therefore,

Correspondence to: Professor Yihe Hu, Department of Orthopedics, Xiangya Hospital of Central South University, 87 Xiangya Road, Changsha, Hunan 410008, P.R. China

E-mail: csuhuyihe@163.com
\end{abstract}

Key words: chondrosarcoma, resveratrol, proliferation, apoptosis, invasion the identification of novel preventive drugs of chondrosarcoma is urgently required.

Resveratrol (Res) is a natural polyphenol compound isolated from various fruits, including grapes, and has been found to be a modulator of cell phenotype with a complex and pleiotropic mode of action (3). Accumulating evidence regarding its activity reveals that Res has an effect on cell proliferation, differentiation, apoptosis and autophagy $(4,5)$. Furthermore, it has been demonstrated that Res has a suppressive effect on various types of human malignancy (6). For instance, Yang et al (7) reported that Res inhibited the growth of gastric cancer by inducing G1 phase arrest and senescence. More recently, the effect of Res on chondrosarcoma has been suggested. Gweon et al (8) found that Res had an inhibitory effect on matrix metalloproteinase-induced differentiation via the p38 kinase and JNK pathways in chondrosarcoma cells. In addition, Im et al (9) showed that Res selectively compromised the survival of human chondrosarcoma cells. However, the detailed effect of Res on chondrosarcoma as well as the underlying molecular mechanisms remain largely unclear.

The present study aimed to investigate the effects of Res on cell proliferation, apoptosis and invasion in chondrosarcoma cells. In addition, the underlying molecular mechanisms involved were also analyzed.

\section{Materials and methods}

Reagents. Res and MTT were purchased from Sigma-Aldrich (St. Louis, MO, USA), which was dissolved in dimethyl sulfoxide (Sigma-Aldrich, St. Louis, MO, USA) to make a $500 \mathrm{mM}$ stock solution and stored at $-80^{\circ} \mathrm{C}$.

Cell culture. JJ012 and SW1353 human chondrosarcoma cell lines were purchased from Nlunbio (Changsha, China). Cells were cultured in RPMI-1640 medium (Life Technologies, Carlsbad, CA, USA) with $10 \%$ fetal bovine serum at $37^{\circ} \mathrm{C}$ in a humidified incubator containing $5 \% \mathrm{CO}_{2}$. After culturing for $48 \mathrm{~h}$, cells were serum starved for $24 \mathrm{~h}$, and then treated with or without Res. The present study was approved by the ethics committee of Xiangya Hospital of Central South University (Changsha, China).

MTT assay. Cell viability was determined using MTT, according to the manufacturer's instructions. In brief, cells 
were suspended in $0.2 \mathrm{ml}$ medium at a concentration of 5,000 cells/well and incubated overnight in 96-well plates. Absorbance at $490 \mathrm{~nm}$ was measured using a microplate reader (Molecular Devices, Sunnyvale, CA, USA).

Apoptosis analysis. The Annexin V-fluorescein isothiocyanate Apoptosis Detection kit (Sigma-Aldrich) was used to perform cell apoptosis analysis. For each group, 106 cells were resuspended in binding buffer, and then stained with $2.5 \mu 1$ Annexin V-FITC and $5 \mu 1$ propidium iodide for $15 \mathrm{~min}$ in the dark. Then, cells were then washed with Dulbecco's phosphate-buffered saline and analyzed by flow cytometry (C6; BD Biosciences, Franklin Lakes, NJ, USA).

Cell invasion assay. The cell invasive ability was investigated using 24-well Transwell chambers (Chemicon, USA) with a layer of Matrigel (BD Biosciences). According to the manufacturer's instructions, $300 \mu 1$ cell suspension $\left(10^{6}\right.$ cells $\left./ \mathrm{ml}\right)$ was added into the upper chamber, and $500 \mu \mathrm{l}$ RPMI-1640 with $10 \%$ fetal bovine serum was added into the lower chamber. After incubation for $24 \mathrm{~h}$, cells on the lower surface of the membrane was stained, rinsed and dried in air. Five fields were randomly selected and the cell number was counted under a microscope (CX22; Olympus, Tokyo, Japan).

Western blotting. Tissues or cells were solubilized in cold radioimmunoprecipitation assay lysis buffer (Sigma-Aldrich). Proteins were separated with $12 \%$ SDS-PAGE, and transferred onto a polyvinylidene difluoride (PVDF) membrane (Life Technologies), which was then incubated with Trisbuffered saline with Tween-20 (Sigma-Aldrich) containing $5 \%$ milk at room temperature for $3 \mathrm{~h}$. The PVDF membrane was then incubated with rabbit monoclonal anti-matrix metalloproteinase (MMP)2 (cat. no. ab51125), rabbit polyclonal MMP9 (cat. no. ab38898), rabbit polyclonal phosphorylated (p)-phosphoinositide 3-kinase (PI3K; cat. no. ab182651), rabbit monoclonal PI3K (cat. no. EP380Y), rabbit polyclonal p-AKT (cat. no ab66138), rabbit monoclonal AKT (cat. no. ab32505), rabbit polyclonal p-p38 mitogen-activated protein kinase (MAPK; cat. no. ab47363), rabbit polyclonal p38 MAPK (cat. no. ab7952) and rabbit polyclonal GAPDH (cat. no. ab181602) primary antibodies (Abcam, Cambridge, UK), at room temperature for $3 \mathrm{~h}$. After washing with phosphate-buffered saline with Tween-20 three times, the membrane was incubated with the mouse anti-rabbit secondary antibodies (Abcam) at room temperature for $40 \mathrm{~min}$. Chemiluminescent detection was performed using an enhanced chemiluminescence kit (Pierce Chemical, Rockford, IL, USA). The relative protein expression was analyzed by Image-Pro plus software 6.0 (Media Cybernetics, Rockville, MD, USA).

Statistical analysis. All data are presented as the mean \pm standard deviation. Comparisons between the two groups were determined by a two-tailed Student's t-test using the Graph-Pad Prism 4 program (GraphPad Software, San Diego, CA, USA). Analysis of variance was used to assess comparisons between multiple groups. $\mathrm{P}<0.05$ was considered to indicate a statistically significant difference.
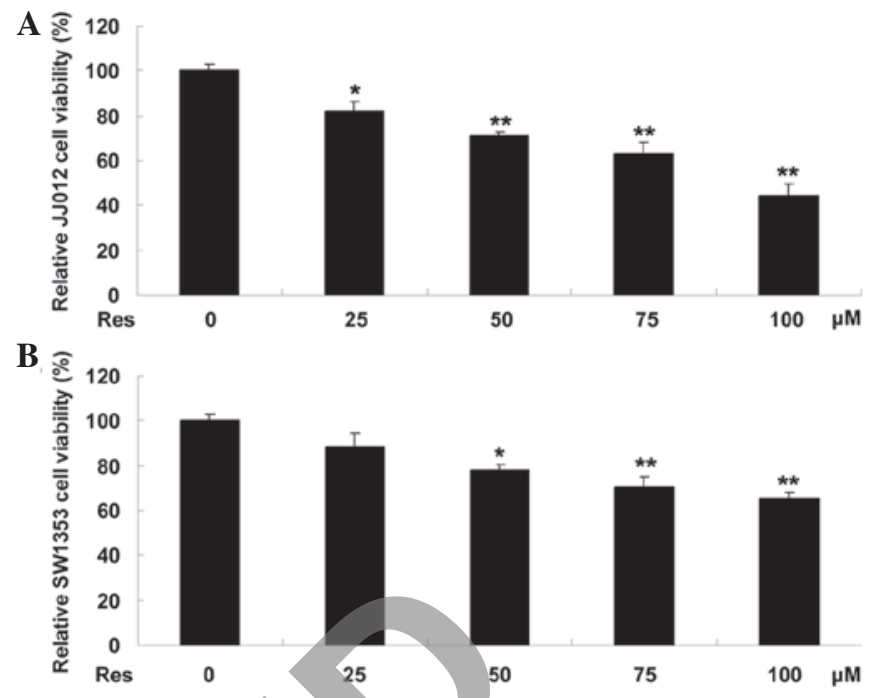

Figure 1. An MTT assay was performed to determine the effect of Res on cell proliferation in (A) JJ012 (B) SW1353 chondrosarcoma cells, which were treated with Res $(0-100 \mu \mathrm{M})$ for $24 \mathrm{~h}$. ${ }^{*} \mathrm{P}<0.05$ and ${ }^{* *} \mathrm{P}<0.01$ vs. $0 \mu \mathrm{M}$.

\section{Results}

Res inhibits chondrosarcoma cell proliferation. It was first hypothesized that Res can suppress chondrosarcoma cell proliferation. To test this hypothesis, the effect of Res on chondrosarcoma cell growth was investigated. As demonstrated in Fig. 1A, Res inhibited JJ012 cell growth in a dose-dependent manner. Following treatment with Res (25-100 $\mu \mathrm{M})$ for $24 \mathrm{~h}$, the growth speed of JJ012 cells was notably decreased from $82.4 \%$ to $44.3 \%$, compared with the untreated control JJ012 cells. A similar effect was also found in the SW1353 chondrosarcoma cell line. As shown in Fig. 1B, following treatment with Res $(25-100 \mu \mathrm{M})$ for $24 \mathrm{~h}$, the proliferation rate of SW1353 cells was decreased from 88.5 to $65.3 \%$. In addition, since the inhibitory effect of Res on JJ012 cells was greater, JJ012 cells were used in the subsequent experiments.

Res induces apoptosis in chondrosarcoma cells. The effect of Res on JJ012 chondrosarcoma cell apoptosis was then investi gated. As shown in Fig. 2, the cell apoptosis level was notably upregulated after treatment with Res in JJ012 chondrosarcoma cells in a dose-dependent manner. Following treatment with Res $(25-100 \mu \mathrm{M})$ for $24 \mathrm{~h}$, the relative apoptosis rate of JJ012 cells was notably increased from 3.11 to $11.73 \%$, compared with the untreated control JJ012 cells. These findings indicated that Res induced JJ012 chondrosarcoma cell apoptosis.

Res exhibits suppressive effects on chondrosarcoma cell invasion. The effect of Res on JJ012 chondrosarcoma cell invasion was then investigated. As shown in Fig. 3A, following treatment with Res for $24 \mathrm{~h}$, JJ012 cell invasion was significantly decreased in a dose-dependent manner, compared with the untreated control JJ012 cells. These data suggest that Res has an inhibitory effect on chondrosarcoma cell invasion. Subsequently, the protein level of MMP2 and MMP9 in chondrosarcoma cells was examined with or 

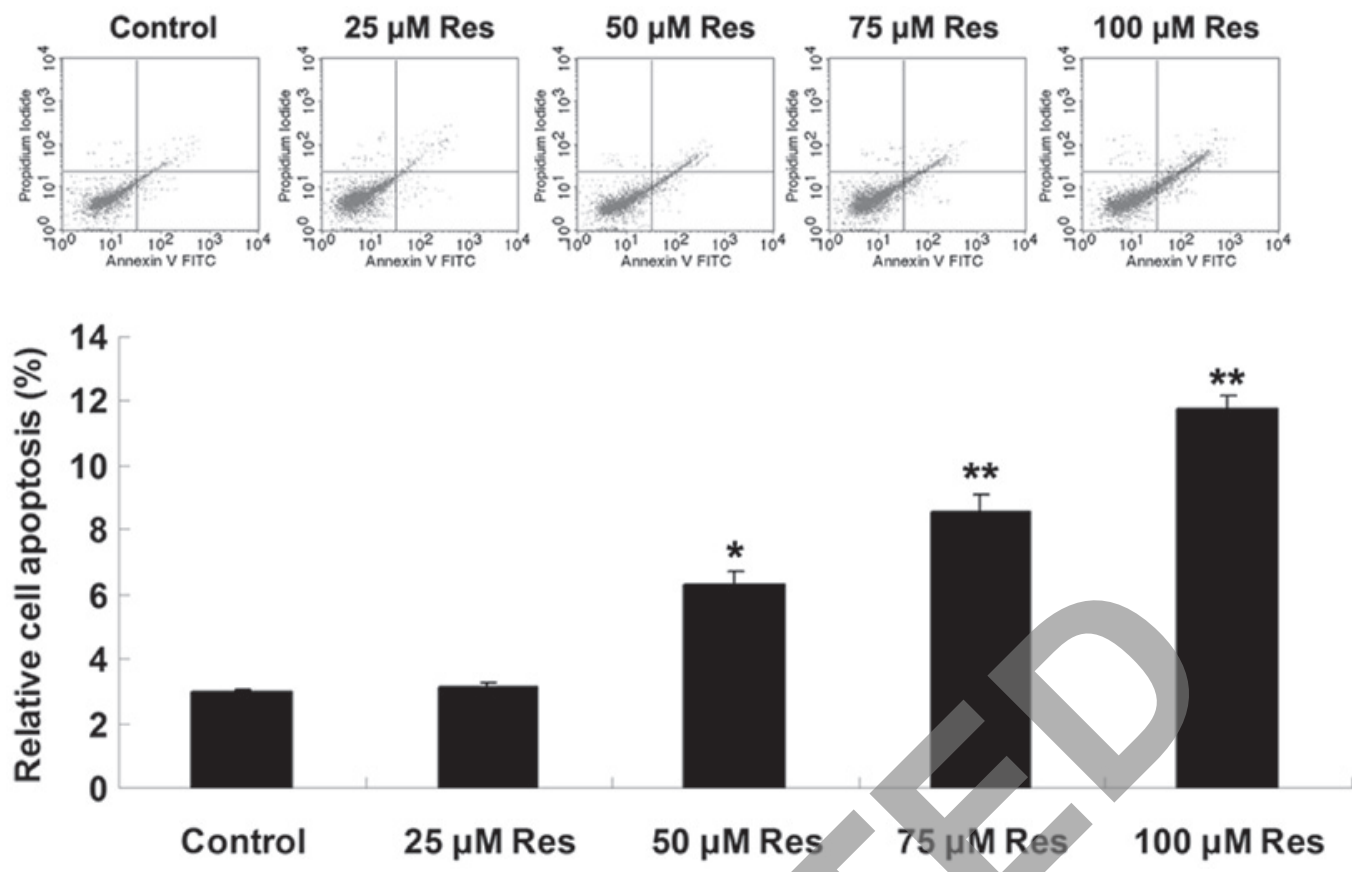

Figure 2. Apoptosis analysis was performed to examine the effect of Res on cell apoptosis in J012 chondrosarcoma cells. Control, JJ012 cells without any treatment. Res, JJ012 cells were treated with 25-100 $\mu \mathrm{M}$ Res for $24 \mathrm{~h} .{ }^{~} \mathrm{P}<0.05$ and $^{* * *} \mathrm{P}<0.01$ vs. control. Res, resveratrol.

A
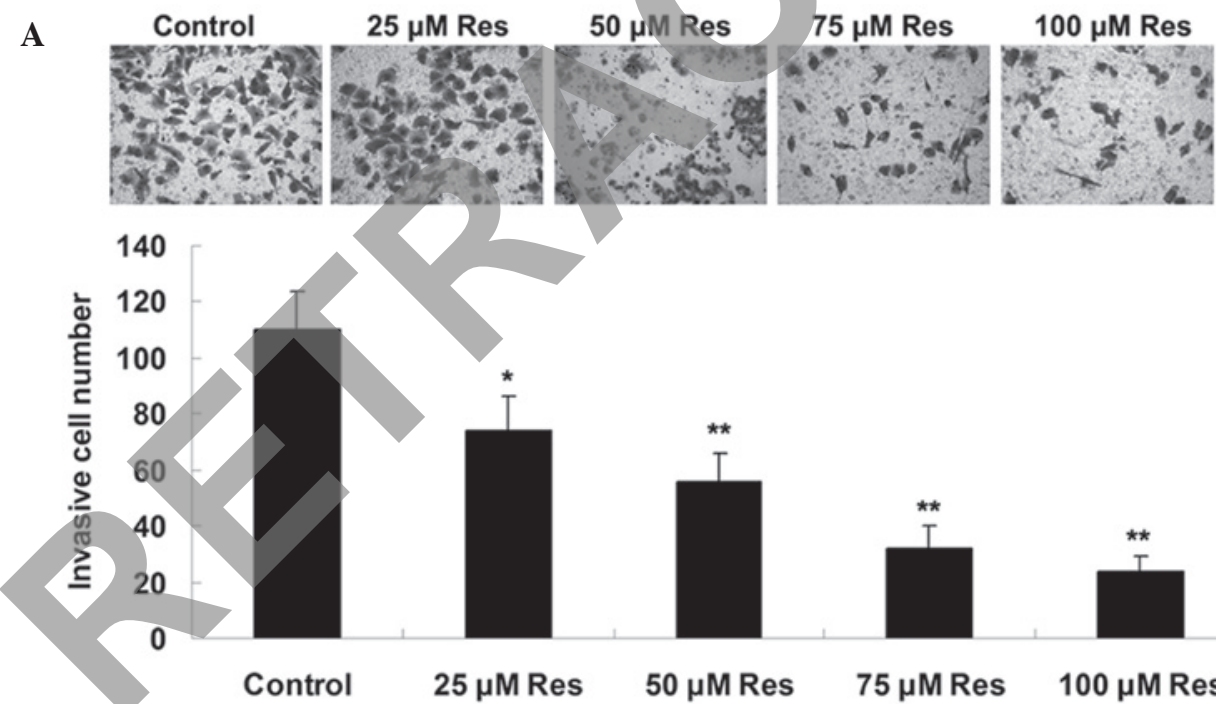

B

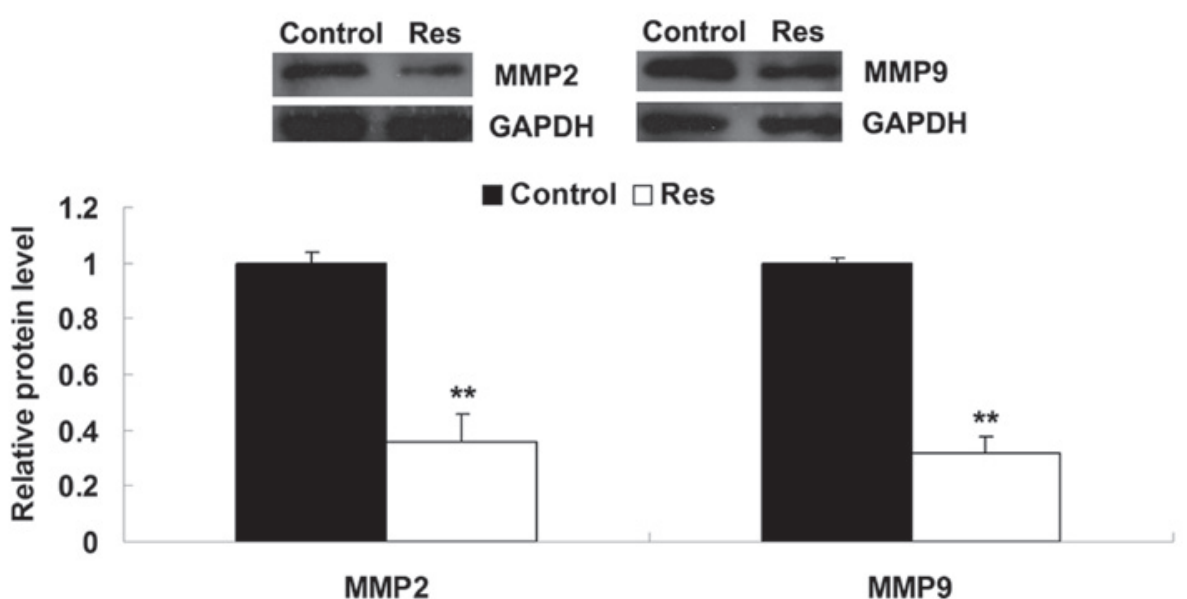

Figure 3. (A) A Transwell assay was performed to determine the effect of Res on cell invasion of JJ012 chondrosarcoma cells. (B) Western blot analysis was performed to determine the protein level of MMP2 and MMP9. GAPDH was used as an internal reference. Control, JJ012 cells without any treatment. Res, JJ012 cells were treated with $75 \mu \mathrm{M}$ Res for $24 \mathrm{~h}$. ${ }^{*} \mathrm{P}<0.05$ and ${ }^{* *} \mathrm{P}<0.01$ vs. control. Res, resveratrol; MMP, matrix metalloproteinase. 


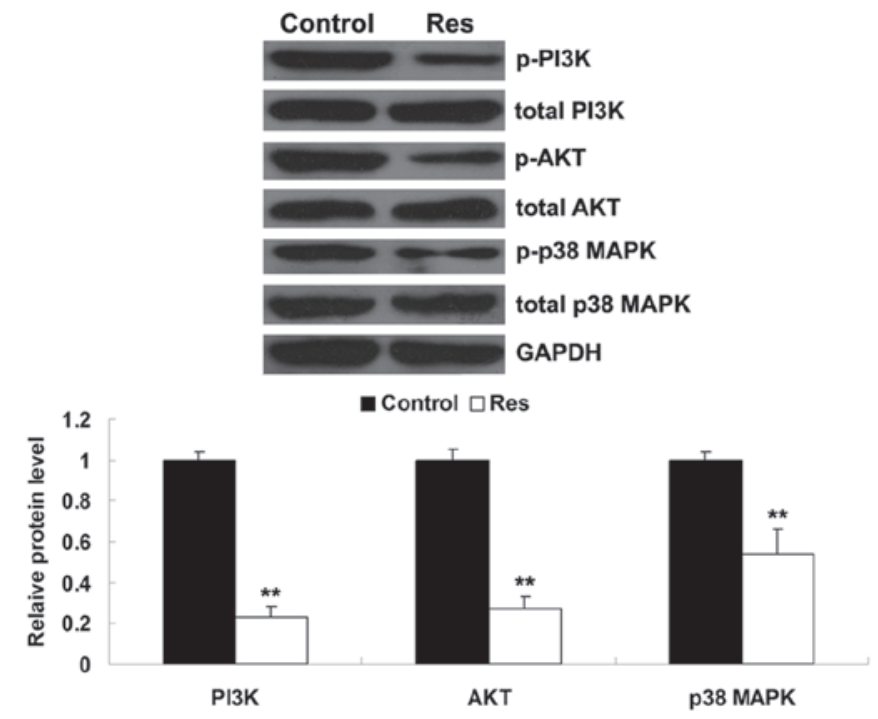

Figure 4. Western blot analysis was performed to determine the p- or total protein level of PI3K, AKT and p38 MAPK, respectively. GAPDH was used as an internal reference. Control, JJ012 cells without any treatment. Res, JJ012 cells were treated with $75 \mu \mathrm{M}$ Res for 24 h. ${ }^{* *} \mathrm{P}<0.01$ vs. control. Res, resveratrol; PI3K, phosphoinositide 3-kinase; MAPK, mitogen-activated protein kinase; p-, phosphorylated.

without treatment with $75 \mu \mathrm{M}$ Res. The data showed that treatment with Res caused a decrease in the protein levels of MMP2 and MMP9 in chondrosarcoma cells, suggesting that MMP2 and MMP9 were involved in Res-induced inhibition of chondrosarcoma cell invasion (Fig. 3B).

Res inhibits the activity of PI3K and MAPK signaling pathways in chondrosarcoma cells. As PI3K and MAPK signaling pathways have been demonstrated to be involved in the regulation of proliferation, apoptosis and invasion in various types of cancer (10), the effect of Res on the activity of PI3K and MAPK signaling pathways was investigated. As demonstrated in Fig. 4, the data showed that the activity of PI3K, AKT and p38 MAPK was significantly downregulated following treatment with $75 \mu \mathrm{M}$ Res for $24 \mathrm{~h}$ in chondrosarcoma cells, compared with untreated control cells. The results suggest that PI3K and MAPK signaling pathways may be involved in the effects of Res on cell proliferation, apoptosis and invasion in chondrosarcoma cells.

\section{Discussion}

Res is a natural polyphenol and has been demonstrated to act as a promising antitumor agent (6). Although Res has been shown to have suppressive effects on several types of human malignancies in vitro and in vivo, its chemotoxic effect on chondrosarcoma cells as well as the molecular mechanism remains largely unclear. In the present study, it was identified that Res inhibited chondrosarcoma cell proliferation and the antiproliferative activity of Res occurred in a dose-dependent manner. In addition, Res could significantly induce chondrosarcoma cell apoptosis. Furthermore, Res was also found to suppress chondrosarcoma cell migration and invasion. Molecular mechanism investigation revealed that PI3K and MAPK signaling pathways were involved in
Res-induced inhibition of proliferation, migration and invasion in chondrosarcoma cells.

Res has been demonstrated to suppress proliferation but induce apoptosis in multiple types of cancer $(7,13)$. However, the effects of Res on chondrosarcoma cell proliferation and apoptosis remain unknown. The present study found that Res could inhibit proliferation but induce apoptosis in chondrosarcoma cells. The PI3K signaling pathway has been widely investigated in various types of cancer. It is involved in the regulation of a number of intracellular physiological processes, including survival, proliferation, cell cycle progression and migration (11). In addition, Res was also reported to inhibit colon cancer cell proliferation through inhibition of PI3K and $\mathrm{Wnt} / \beta$-catenin signaling pathways (12). Sui et al (13) showed that Res exhibited significant cytotoxic effects and induced apoptosis in K562 chronic myeloid leukemia cells via inhibiting the PI3K signaling pathway. The present study also found that Res had an inhibitory effect on the activity of the PI3K signaling pathway in chondrosarcoma cells, suggesting that inhibition of PI3K signaling may be a common molecular mechanism by which Res suppresses cancer cell proliferation.

Moreover, several studies have reported that Res could inhibit cell inyasion in several types of malignant tumors. For instance, Shan et al (14) reported that Res could inhibit migration, and invasion of oral squamous cell carcinoma cells. Ji et al (15) found that Res suppressed invasion and metastasis of colorectal cancer cells. However, the effect of Res on chondrosarcoma cell invasion has not previously been reported. The present study showed that Res also markedly inhibited chondrosarcoma cell invasion. In addition, it was also shown that the protein levels of MMP2 and MMP9 were also reduced after treatment with Res. As two major members in the MMP protein family, MMP-2 and MMP-9 have been widely investigated in invasion and metastasis in various types of cancer $(16,17)$. Gweon et al (18) showed that Res had an effect on MMP9 in human fibrosarcoma cells. In addition, they also showed that Res attenuated MMP2- and MMP9-regulated differentiation of chondrosarcoma cells through the p38 kinase and JNK pathways (8). Res also inhibited the epithelial-mesenchymal transition of pancreatic cancer cells via mediating the expression of MMP2 and MMP9 (19). These results indicate that Res may inhibit chondrosarcoma cell invasion by inhibiting the expression of MMP2 and MMP9. Furthermore, p38-MAPK was also found to be regulated by Res in chondrosarcoma cells. It has been reported that the MAPK pathway acts as a key regulator in the expression of MMPs in various types of cancer, including chondrosarcoma (20). Res was also reported to inhibit the activity of p38 MAPK signaling. For instance, Res inhibited MAPK activity in coronary artery smooth muscle (21). In addition, Res suppressed mouse skin tumor growth by inhibition of activated p38 MAPK (22). Accordingly, Res may be used for the treatment of chondrosarcoma metastasis.

In conclusion, to the best of our knowledge, this study demonstrated for the first time that Res inhibited cell proliferation and invasion and induced cell apoptosis in chondrosarcoma cells via regulation of PI3K and MAPK signaling pathways. These findings suggest that Res may serve as a promising agent for the treatment of chondrosarcoma. 


\section{Acknowledgements}

This study was supported by the Fundamental Research Funds for the Central Universities of Central South University (grant no. 013zzts092).

\section{References}

1. Mosier SM, Patel T, Strenge K and Mosier AD: Chondrosarcoma in childhood: the radiologic and clinical conundrum. J Radiol Case Rep 6: 32-42, 2012.

2. Logie CI, Walker EA, Forsberg JA, Potter BK and Murphey MD: Chondrosarcoma: A diagnostic imager's guide to decision making and patient management. Semin Musculoskelet Radiol 17: 101-115, 2013.

3. Raederstorff D, Kunz I and Schwager J: Resveratrol, from experimental data to nutritional evidence: the emergence of a new food ingredient. Ann N Y Acad Sci 1290: 136-141, 2013.

4. Borriello A, Bencivenga D, Caldarelli I, et al: Resveratrol: from basic studies to bedside. Cancer Treat Res 159: 167-184, 2014.

5. Widlund AL, Baur JA and Vang O: mTOR: more targets of resveratrol? Expert Rev Mol Med 15: e10, 2013.

6. Singh CK, George J and Ahmad N: Resveratrol-based combinatorial strategies for cancer management. Ann N Y Acad Sci 290: 113-121, 2013

7. Yang Q, Wang B, Zang W, et al: Resveratrol inhibits the growth of gastric cancer by inducing G1 phase arrest and senescence in a Sirt1-dependent manner. PLoS One 8: e70627, 2013.

8. Gweon EJ and Kim SJ: Resveratrol attenuates matrix metalloproteinase-9 and -2-regulated differentiation of HTB94 chondrosarcoma cells through the $\mathrm{p} 38$ kinase and JNK pathways. Oncol Rep 32: 71-78, 2014.

9. Im HJ, Li X, Chen D, et al: Biological effects of the plant-derived polyphenol resveratrol in human articular cartilage and chondrosarcoma cells. J Cell Physiol 227: 3488-3497, 2012.

10. Siegfried Z, Bonomi S, Ghigna $C$ and Karni R: Regulation of the Ras-MAPK and PI3K-mTOR signalling pathways by alternative splicing in cancer. Int J Cell Biol 2013: 568931, 2013.
11. Cantley LC: The phosphoinositide 3-kinase pathway. Science 296: 1655-1657, 2002.

12. Liu YZ, Wu K, Huang J, et al: The PTEN/PI3K/AKT and Wnt/ $\beta$-catenin signaling pathways are involved in the inhibitory effect of resveratrol on human colon cancer cell proliferation. Int J Oncol 45: 104-112, 2014.

13. Sui T, Ma L, Bai X, Li Q and Xu X: Resveratrol inhibits the phosphatidylinositide 3-kinase/protein kinase $\mathrm{B} /$ mammalian target of rapamycin signaling pathway in the human chronic myeloid leukemia K562 cell line. Oncol Lett 7: 2093-2098, 2014.

14. Shan Z, Yang G, Xiang W, Pei-jun W and Bin Z: Effects of resveratrol on oral squamous cell carcinoma (OSCC) cells in vitro. J Cancer Res Clin Oncol 140: 371-374, 2014.

15. Ji Q, Liu X, Fu X, et al: Resveratrol inhibits invasion and metastasis of colorectal cancer cells via MALAT1 mediated Wnt/ $\beta$-catenin signal pathway. PLoS One 8: e78700, 2013.

16. Poincloux R, Lizárraga $\mathrm{F}$ and Chavrier P: Matrix invasion by tumour cells: a focus on MT1-MMP trafficking to invadopodia. J Cell Sci 122: 3015-3024, 2009

17. Turpeenniemi-Hujanen T: Gelatinases (MMP-2 and -9) and their natural inhibitors as prognostic indicators in solid cancers. Biochimie 87: 287-297, 2005.

18. Gweon EJ and Kim SJ: Resveratrol induces MMP-9 and cell migration via the $\mathrm{p} 38$ kinase and PI-3 K pathways in HT1080 human fibrosarcoma cells. Oncol Rep 29: 826-834, 2013.

19. Li W, Ma J, Ma Q, et al: Resveratrol inhibits the epithelial-mesenchymal transition of pancreatic cancer cells via suppression of the PI-3K/AKT/NF-кB pathway. Curr Med Chem 20: 4185-4194, 2013.

20. Pei Y, Harvey A, Yu XP, Chandrasekhar S and Thirunavukkarasu K: Differential regulation of cytokine-induced MMP-1 and MMP-13 expression by $\mathrm{p} 38$ kinase inhibitors in human chondrosarcoma cells: potential role of Runx 2 in mediating p38 effects. Osteoarthritis Cartilage 14: 749-758, 2006

21. El-Mowafy AM and White RE: Resveratrol inhibits MAPK activity and nuclear translocation in coronary artery smooth muscle: reversal of endothelin-1 stimulatory effects. FEBS Lett 451: 63-67, 1999.

22. George J, Singh M, Srivastava AK, et al: Resveratrol and black tea polyphenol combination synergistically suppress mouse skin tumors growth by inhibition of activated MAPKs and p53. PLoS One 6: e23395, 2011. 